ISSN 1112-9867

\title{
THE RELIABILITY OF DATA PRODUCED BY ISOKINETIC DYNAMOMETRY (IKD) OF KNEE EXTENSION AND FLEXION MOVEMENT
}

\author{
Z. A. Malik ${ }^{1,2, *}$, J. Vanrenterghem ${ }^{2}$ and J. G. Burniston ${ }^{2}$ \\ ${ }^{1}$ Faculty of Sports Science and Coaching, Universiti Pendidikan Sultan Idris, Tg Malim, \\ Perak, Malaysia \\ ${ }^{2}$ Research Institute for Sport \& Exercise Sciences, Liverpool John Moores University, \\ Liverpool, UK
}

Published online: 10 November 2017

\begin{abstract}
To investigate the feasibility of using isolated knee performance to measure the f-v profile of human muscle in vivo, we were using isokinetic dynamometry (IKD) technique that span the entire f-v profile of skeletal muscle. With institutional ethics approval, eleven healthy males (mean \pm SD: age, $24.9 \pm 3.1$ years; body mass, $80.2 \pm 10.1 \mathrm{~kg}$; height, $176.2 \pm 7.1 \mathrm{~cm}$ ) completed this study. The participants were physically active and were familiarized with the techniques and protocol prior to completing 4 experimental sessions (1 introductory session and 3 repeated experiment sessions). The sessions was used a protocol of knee extensor function on IKD at isometric mode and angular velocities of $60^{\circ} . \mathrm{s}^{-1}, 120^{\circ} . \mathrm{s}^{-1}, 240^{\circ} . \mathrm{s}^{-1}$ and $450^{\circ} . \mathrm{s}^{-1}$. IKD data were not significantly different across the 3 repeated trials, demonstrating there was no evidence of systematic bias. The average coefficient of variation (CV) of IKD was $9.3 \pm 3.0 \%$ ranging from 7.0 to $15.7 \%$. Therefore the isolated knee performance to measure the f-v profile of human muscle in vivo using the IKD was feasible.
\end{abstract}

Author Correspondence, e-mail: zulezwan@fsskj.upsi.edu.my

doi: http://dx.doi.org/10.4314/jfas.v9i6s.90 
Keywords: f-v profile, isokinetic dynamometry, skeletal muscle

\section{INTRODUCTION}

\subsection{Techniques of the knee extensors}

There are various techniques that can be implement to test the muscles capability to produce force with difference speed of contraction. Isokinetic testing can be used to investigate power output and also differences in the force-velocity profile of muscle groups. Isokinetic dynamometers control the velocity of joint flexion or extension by applying variable resistance throughout the range of motion. Therefore the velocity of joint rotation is constant, excluding acceleration to and deceleration from the designated velocity and the force is dependent on how hard the individual pushes against the load cell [1]. IKD can be used at low, moderate and high velocities depending on the different evaluations or programs and provides reliable data. Data acquisition and analysis have been improved by using computer systems interfaced to isokinetic dynamometers. Recently developed computer systems provide correction for gravitational and inertial errors, accurate computation of isokinetic parameters and real-time display of the torque output.

Isokinetic muscle contractions at velocities of 60,180 and $300 \mathrm{deg} / \mathrm{s}$ and investigated difference in force output of weight lifters compared to untrained participants [2]. This study was designed to determine velocity-specific isokinetic forces and cross sectional areas of reciprocal muscle groups measured by B-mode ultrasonic apparatus in weight lifters $(n=34)$ and untrained male participants $(n=31)$. Force declined at higher velocity movements (as expected from muscle force-velocity curve) in both sedentary individuals and Olympic weight lifters. However, this effect was less pronounced in Olympic weight lifters and this trend may be due to adaptations induced by their training regimen, which places emphasis on high velocity movements. These findings suggest isokinetic dynamometry could be used to study the force velocity profile of human muscle groups in vivo but first we wanted to confirm that a commercial isokinetic dynamometer was able to measure the entire range of velocities produced during knee extension. 


\section{Aim of this study}

We conducted force-velocity and power-velocity analyses of isolated knee exercise using standard isometric and IKD and test the reliability at these measurements. If these are found to be reliable we can use this protocol as the main physiological assessment in subsequent studies.

\section{EXPERIMENTAL}

\section{Measurement on isokinetic dynamometry}

\subsection{Participants}

Eleven male participants with different sporting backgrounds (e.g sprinter, rugby player, footballer etc.) participated in this study, which was approved by LJMU ethics committee reference number $12 / \mathrm{SPS} / 013$. The mean $( \pm \mathrm{SD})$ age, height, body mass were $24.9 \pm 3.1$ years, $176.2 \pm 7.1 \mathrm{~cm}$ and $80.2 \pm 10.1 \mathrm{~kg}$, respectively. Participants were questioned on their training history and the Physical Readiness Questionnaire (PAR-Q; [3]) was used as a screening tool to assess each volunteer's level of physical fitness and ability to engage in physical activity. Each participant was required to perform 4 sessions (1 introductory session and 3 repeated experiment sessions). The session were used isokinetic dynamometry of knee extension movement at 5 different velocities. All measurements were performed at the same time of day for each participant to limit diurnal variation [4].

\subsection{Isokinetic dynamometry}

IKD was performed at different velocities to investigate the reliability of data across f-v curve. Knee extension and flexion performance was measured according to standard protocols using an isokinetic dynamometer (Multi-Joint System 3 Pro; Biodex Medical Systems, New York, USA). Strict adherence to the guidelines of the Biodex isokinetic dynamometer operations manual was followed. Participants were familiarized with use of the dynamometer and testing procedures prior to the baseline measurements to reduce possible influences of test habituation on muscular performance. Each participant completed a warm-up protocol prior to analysis of their muscular performance. The warm-up session include 5 minutes cycling on a cycle ergometer (Monark Ergomedic 824E) set with $150 \mathrm{~W}$ resistance and a standardise 
routine of quadriceps and hamstrings stretching exercises. After a 5 minutes rest on completion of the warm-up, the participants were instructed to continue with the testing procedure.

The participant was seated; leaning against a backrest inclined at $16^{\circ}$ from vertical and with the seat inclined $6^{\circ}$ from horizontal. The axis of the knee was aligned with the axis of the Biodex dynamometer exercise arm. The accuracy of alignment was checked by allowing the participant to extend the leg while pushing against the shin pad that was positioned over the lower third of the leg. If the pad did not move up or down the leg over the range of motion to be tested, the knee was considered to be aligned with the axis of the lever arm.

The participant's position was secured using thigh, pelvic and torso straps in order to minimize body movements during the test procedure. Shoulder straps were applied diagonally across the chest with the participant's arms crossed and their palms on opposite shoulders to minimize excessive upper body movement and muscular substitution. The lateral femoral epicondyle was used as a bony landmark for matching the axis rotation of the knee joint and the axis rotation of the dynamometer shaft.

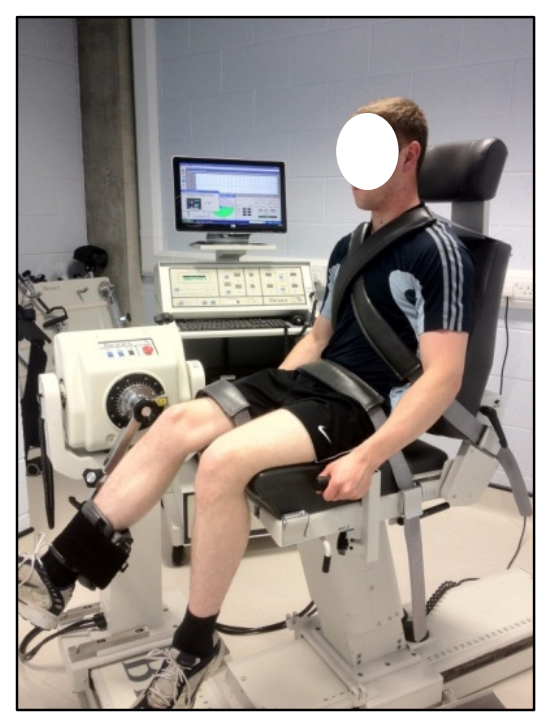

Fig.1. The participant was seated and position was secured on IKD

Each participant used the same radius for each test but the angular velocities were tested in a different order for each session using balanced Latin Square design. Test angular velocities were set at $60^{\circ} . \mathrm{s}^{-1}, 120^{\circ} . \mathrm{s}^{-1}, 240^{\circ} . \mathrm{s}^{-1}$ and $450^{\circ} . \mathrm{s}^{-1}$. Participants were asked to do 3 repetitions of extension and flexion for each angular velocity. One minute rest was given between each 
angular velocity. Peak torque (PT) and average power (AV) were recorded and exported as an excel file for further analysis through the report after the testing.

After completing the isokinetic test, participants were asked to remain at the same position and rest for 5 minutes. Meanwhile the protocol was changed to isometric mode. The participants performed isometric contractions at a static knee joint angle at $60^{\circ}$. They were instructed to extend their knee "as fast and hard as possible" and sustain the maximal voluntary isometric contraction for two seconds. The participants were asked to do 3 repetitions with 60 seconds rest between each attempt.

\subsection{Data analysis}

The data were analysed using Statistical Package for Social Sciences (SPSS) version 17.0 software (SPAA v.17, SPSS Inc, Chicago). To assess reliability across the three testing, one way ANOVA analysis repeated measures was used to analyse the main effects of time. Bonferroni test for multiple comparisons was used to determine the differences when one way ANOVA repeated measure showed a significant effect of time. Significance level was set at $<0.05$, and all data were presented as mean + SD. Furthermore we look at the reproducibility. Typically reproducibility is expressed as a coefficient variation (CV) relative to measurement value. There are various methods of calculating $\mathrm{CV}$, the simplest method is with data from repeated measurements on a single case, where the standard deviation (SD) of the error is divided by the total mean and multiplied by 100 [5].

\section{RESULTS AND DISCUSSION}

Test re-test reliability of IKD measurements was assessed using repeated measures ANOVA and the coefficient of variation (CV) was calculated for major parameters. IKD data (Table 1) were not significantly different across the 3 repeated trials, demonstrating there was no evidence of systematic bias. Moreover, post-hoc analysis comparing trials 1-2, 1-3 and 2-3 did not reveal significant random effects. The average CV of IKD parameters was $9.38 \pm 3.0 \%$ ranging from 7.0 to $15.7 \%$. 
Table 1. Reliability of muscle performance test.

\begin{tabular}{llll}
\hline & Mean & $\begin{array}{l}\text { ANOVA } \\
(\mathbf{p}) \text { value }\end{array}$ & $\begin{array}{l}\text { \% Coefficient of } \\
\text { Variation }(\mathbf{C V})\end{array}$ \\
\hline IKD Peak Torque(Nm) & & 7.9 \\
Isometric & $251.22 \pm 50.59$ & 0.273 & 9.2 \\
60 & $222.95 \pm 40.91$ & 0.181 & 7.0 \\
120 & $195.43 \pm 41.95$ & 0.322 & 8.4 \\
240 & $146.39 \pm 28.68$ & 0.087 & 6.4 \\
450 & $109.17 \pm 17.46$ & 0.213 & 12.7 \\
IKD Average Power(W) & & & 7.2 \\
60 & $149.87 \pm 32.44$ & 0.337 & 15.7 \\
120 & $251.74 \pm 58.02$ & 0.308 & 9.9 \\
240 & $331.22 \pm 77.55$ & 0.240 & 0.269 \\
450 & $357.03 \pm 83.72$ & 0.269 & \\
\hline
\end{tabular}

Data are presented as means $\pm \mathrm{SD} ; n=11$ participants averaged from 3 separate test sessions. Isokinetic dynamometry (IKD) was performed at 5 different velocities: 0 (isometric), 60, 120, 240 and $450^{\circ} . \mathrm{s}^{-1}$. Data presented are peak torque $(\mathrm{Nm})$ and average power $(\mathrm{W})$ over 3 kicks during knee extension.

The overall aim of this study was to investigate the reliability of data produced by IKD of knee extension and flexion movement. To assess the reliability of IKD data, ANOVA with repeated measures including a correction for 'sphericity' [5] was used for assessing systematic bias across repeated [5,6]. Using appropriate post hoc multiple comparisons, random bias between individual tests was also assessed. Participants performed the isokinetic dynamometry testing at 5 different velocities at $0^{\circ} . \mathrm{s}^{-1}, 60^{\circ} . \mathrm{s}^{-1}, 120^{\circ} . \mathrm{s}^{-1}, 240^{\circ} . \mathrm{s}^{-1}$ and $450^{\circ} . \mathrm{s}^{-1}$. In the present study, after 3 repeated sessions of the isokinetic dynamometry testing, one way ANOVA for repeated measures revealed that there was no significant difference across the 3 sessions for any of the performance variables that were recorded.

Typically reproducibility is expressed as a coefficient variation $(\mathrm{CV})$ relative to measurement value. There are various methods of calculating $\mathrm{CV}$, the simplest method is with data from repeated measurements on a single case, where the standard deviation (SD) of the error is 
divided by the total mean and multiplied by 100 [7]. The use of CV assumes that the largest test-retest variation occurs in the individuals scoring the highest values on the test [8]. Some scientists have arbitrarily chosen an analytical goal of the CV being $10 \%$ or less [9]. This does not mean that all variability between tests is always less than $10 \%$ of the mean. In the current work, percentage of $\mathrm{CV}$ was below $10 \%$ except on $60^{\circ} . \mathrm{s}^{-1}(12.7 \%)$ and $240^{\circ} . \mathrm{s}^{-1}(15.7 \%)$ isokinetic knee extension average power (W). A study on reliability of measures obtained during countermovement jumps that all CV were below 10\% [10]. In most parameters in this study showed no significant different in $\mathrm{CV} \%$ with current study, for instance peak torque $(\mathrm{Nm})$ and avarage power $(\mathrm{W})$.

\section{CONCLUSION}

We investigated the reliability of isolated knee performance measures across the f-v profile of human muscle encompassing a range of velocities using isokinetic dynamometry (IKD). The reliability of the measure of knee extensor performance was acceptable $(<15 \% \mathrm{CV})$.

\section{ACKNOWLEDGEMENTS}

Z.A.Malik was funded by UPSI and Ministry of Higher Education, Malaysia.

\section{REFERENCES}

[1] Huang, M.H., Lin, Y.S., R.C. \& Lee, C.L. (2003). A Comparison of Various Therapeutic Exercises on the functional Status of Patients with Knee Osteoarthritis. Semin Arthritis Rheum, 32, 398-406.

doi: 10.1053/sarh.2003.50021.

[2] Kanehisa, H., \& Fukunaga, T. (1999). Profiles of musculoskeletal development in limbs of college Olympic weightlifters and wrestlers. Eur J Appl Physiol Occup Physiol, 79(5), 414-420. doi: https://doi.org/10.1007/s004210050531

[3] Thomas, S., Reading, J., \& Shephard, R. J. (1992). Revision of the Physical-Activity Readiness Questionnaire (PAR-Q). Canadian Journal of Sport Sciences, 17, 338-345.

[4] Reilly, T. \& Waterhouse, J. (2009). Sports performance: is there evidence that the body clock plays a role? European Journal of Applied Physiology, 106, 321-332. 
doi: 10.1007/s00421-009-1066-x

[5] Vincent, J. (1994). Statistics in kinesiology. Champaign (IL): Human Kinetics.

[6] Thomas J.R. \& Nelson J.K. (1990). Research methods in physical activity. Champaign (IL): Human Kinetics.

[7] Sale D.G. (1991). Testing strength and power. In: MacDougall JD, Wenger HA, Green HJ, editors. Physiological testing of the high performance athlete. Champaign (IL): Human Kinetics, 21-106.

[8] Bland J.M. \& Altman D.G. (1995). Comparing two methods of clinical measurement: a personal history. Int J Epidemiol, 24, 7-14.

[9] Stokes M. (1985). Reliability and repeatability of methods for measuring muscle in physiotherapy. Physiother Pract, 1, 71-6.

[10] Cormack, S. J., Newton, R. U., McGuigan, M. R., \& Doyle, T. L. (2008). Reliability of measures obtained during single and repeated countermovement jumps. International Journal of Sports Physiology and Performance, 3, 131-144

\section{How to cite this article:}

Z. A. Malik, Vanrenterghem J, Burniston J G. The Reliability of Data Produced by Isokinetic Dynamometry (IKD) of Knee Extension and Flexion Movement. J. Fundam. Appl. Sci., 2017, 9(6S), 1219-1226. 